\title{
Updated Palliative Chemotherapy for Biliary Tract Cancer
}

\author{
Jun Ho Ji ${ }^{1}$ and Jung-Hun Kang ${ }^{2 *}$ \\ ${ }^{1}$ Division of Hematology and Oncology, Department of Internal Medicine, Sungkyunkwan University Scholl of Medicine, South Korea \\ ${ }^{2}$ Division of Hematology-Oncology, Department of Internal Medicine, Gyeongsang National University Hospital, South Korea
}

Received: January 24, 2018; Published: February 06, 2018

*Corresponding author: Jung-Hun Kang, Department of Internal Medicine, Gyeongsang National University College of Medicine, 79 Gangnam-ro, S-Korea, Email: junofanclub@hanmail.net

\begin{abstract}
Biliary tract cancer (BTC) is rare and often diagnosed at an advanced stage. Thus, palliative treatment is usually the only option for patients with advanced biliary tract cancer. Many chemotherapeutic agents have been tested. However, few therapeutic medications have been proven to be useful. With the recent increase in the understanding of drug resistance mechanisms and molecular features of cancer cells, possible new drugs are emerging. Herein, we discuss issues including the choice of chemotherapy and optimal chemotherapy and categorizing treatment types into cytotoxic chemotherapy, targeted therapy, and immunotherapy.
\end{abstract}

Keywords: Biliary Tract Cancer; Palliative Chemotherapy

\section{Introduction}

\section{Chemotherapy versus supportive care}

Biliary tract cancer (BTC) includes intrahepatic cholangiocarcinoma, extrahepaticcholangiocarcinoma, gallbladder cancer, and ampulla of Vater cancer. However, advanced BTC shows very poor prognosis regardless of primary location. Although most guidelines endorse chemotherapy for advanced BTC, the benefit of chemotherapy in the aspect of survival and quality-of-life must be proven comparing patients with only best supportive care (BSC [1-3]. The natural course of patients with BTC receiving BSC is reported as an overall survival (OS) of 7.1 months. We reported that chemotherapy improved the OS of patients with advanced BTC by comparing BSC groups (12.7 vs. 7.0 months $(\mathrm{p}<0.001)$ ).[4]The survival benefit was not proven in groups with normal serum cancer antigen (CA) 19-9 levels. Data regarding the benefit of chemotherapy has been consistently reported in other studies $[2,5,6]$. Based on these studies, chemotherapy is justified for advanced BTC cancer.

\section{Cytotoxic chemotherapy}

Until the early 2000s, most studies of palliative cytotoxic chemotherapy for advanced BTC were conducted based on 5-fluorouracil, taxane, and anthracycline, with a response rate of $10-40 \%$ and a survival time of 5-12 month [7]. By the 2000s, most studies for pancreato-biliary cancer had focused on gemcitabine, owing to its widespread use. The ABC-02 trial was the first successful prospective phase III study; combination chemotherapy of cisplatin plus gemcitabine became the standard regimen for initial treatment of advanced BTC [5]. This study demonstrated superior response rates (36\% versus $16 \%)$ and prolonged survival (11.7 months versus 8.1 months) in the combination cohort in comparison with the gemcitabine-alone cohort. Another combination chemotherapy regimen of gemcitabine plus oxaliplatin (GEMOX) or gemcitabine plus capecitabine (GEMCAP) also demonstrated notable outcomes of response rate and survival time [8-13]. In three previous phase II prospective studies, the GEMCAP chemotherapy regimen was demonstrated to be effective with a response rate of $25 \%$ to $31 \%$ and improvement in OS (12.7 months to 14.0 months) [9-11]. Combination chemotherapy with GEMOX is also effective (relative risk [RR] of $20.5 \%$ to $36 \%$ and OS improved from 8.8 months to 15.4 months) for advanced BTC $[8,12,13]$. As first-line chemotherapy, other regimens with the exception of gemcitabine have also been investigated in several studies, which were conducted with titanium silicate (TS)-1 plus cisplatin (SP) [14,15]. or capecitabine plus cisplatin (XP) $[16,17]$. And showed similar results to the standard regimen of gemcitabine plus cisplatin.

Nevertheless, there is no established standard for patients with advanced BTC who are not easy to treat using chemotherapy for reasons including poor performance status, old age, or renal failure. Due to the various prognoses of BTC, some patients with BTC live longer than expected without receiving any treatment. Several retrospective studies about the natural course of BTC showed that 
urgent cytotoxic chemotherapy might not be beneficial for patients with locally advanced disease or low levels of tumor markers $[4,18]$. The recent advances in supportive care have led to concerns about salvage chemotherapy following first-line chemotherapy of cisplatin plus gemcitabine in some patients with advanced BTC. However, there is no clear second-line regimen, and no large-scaled randomized studies have shown significant efficacy. However, several phase II studies of salvage chemotherapy for advanced BTC have demonstrated promising results [19-21].

\section{Targeted therapy}

For advanced BTC, cytotoxic combination chemotherapy is unsatisfactory with suboptimal outcomes. Further understanding of the molecular characteristics for BTC should result in the development of an effective targeted therapy. However, until now, no effective targeted agents have been approved for BTC. Angiogenesis inhibitors and epidermal growth factor receptor (EGFR) blockers that are used for various cancers have been commonly studied for BTC. A single-arm, phase II study on combination therapy of GEMOX plus bevacizumab as a first-line treatment showed positive results with a median survival of 12.7 months [22]. Similar results were achieved in another phase II trial using GEMCAP plus bevacizumab; the study showed a median progression-free survival (PFS) of 9.1 months and OS of 11.3 months [23]. The combination of vascular endothelial growth factor (VEGF) and EGFR inhibition for BTC was not successful as expected. Lubner et al conducted a single-arm phase II study and treated 49 patients with advanced BTC using erlotinib plus bevacizumab [24]. They reported a response rate of $12.2 \%$ and OS of 9.9 months. Sorafenib, another angiogenesis inhibitor, is a tyrosine kinase inhibitor with multiple targets, such as VEGF receptor (VEGFR), platelet-derived growth factor receptor (PDGFR), BRAF, and CRAF, which has also been studied extensively for BTC. Monotherapy and combination therapy of sorafenib have both shown disappointing results in BTC [25-28]. Gruenberger et al conducted a single-arm phase II study and treated 30 patients with BTC using cetuximab plus GEMOX [29]. They reported encouraging results with a RR of $63 \%$ (complete response (CR) of $10 \%$, partial response (PR) of 53\%) and OS of 15.2 months. Thus, a phase II, randomized, controlled trial was conducted by Malka [30]. However, OS was shorter in the GEMOX plus cetuximab arm with 11.0 months compared to 12.4 months in the GEMOX arm. In a Korean phase III trial, 268 patients with BTC were randomized to receive first-line GEMOX with or without the addition of erlotinib [31]. Additional erlotinib led to improvements in tumor response, but failed to prolong the PFS, which was the primary endpoint. In conclusion, despite several inspiriting studies, EGFR and VEGF inhibition have not been successful for BTC treatment. However, recent molecular advances may facilitate the development of clinical outcomes. Fibroblast growth factor receptor (FGFR) fusion and isocitrate dehydrogenase (IDH) 1/2 mutation in intrahepatic BTC, and human epidermal growth factor receptor (HER)2 amplification and alterations in the EGFR-mitogen activated protein kinase (MAPK)phosphatidylinositol3-kinase (PI3K) pathway in gall bladder (GB) cancer and extrahepatic BTC are currently being investigated in clinical trials.

\section{Immunotherapy}

The causes of BTC, including cholelithiasis, chronic parasite infection, obesity, sclerosing cholangitis, and chronic inflammation, may play an important role in the pathogenesis. Chronic inflammation causes immune cells to release inflammatory cytokines, including tumor necrosis factor (TNF)- $\alpha$, transforming growth factor (TGF)- $\beta$, interferon (IFN)- $\gamma$, interleukin (IL)-6, and IL-8, and these cytokines cause proliferation of the biliary epithelium [32]. Therefore, treatment related to the immune system for BTC is considered to hold great promise. Different mechanisms of immunotherapy have been investigated for BTC that include vaccines and immune checkpoint inhibitors. Presentation of specific antigens in vaccines can lead to the development of memory $\mathrm{T}$ cells that can target cancer cells. It is still too early to judge the efficacy of immunotherapy using vaccines for BTC, but some studies have shown promising results. Although the patients were already heavily treated, the disease control rate was relatively high in the studies [33-35]. Furthermore, the immune checkpoint pathway has been shown to play an important role in immune evasion of tumor cells. The expression of programmed cell death (PD)-1 and PD-ligand (L) 1, a family of immune checkpoints, were confirmed in some cases of BTC [36]. And these results are thought to hold promise that immune checkpoint inhibition will be effective for BTC. In the recent decade, immune checkpoint inhibitors have been one of the most studied agents for the treatment of various cancers. Several studies using immune checkpoint inhibitors are ongoing for BTC and pembrolizumab is the only agent with reported interim results [37]. According to the KEYNOTE-28, treatment of pembrolizumab in previously treated 28 patients with BTC with expression of PD-L1 showed a response rate of $17 \%$ and disease control rate of $34 \%$.

\section{Conclusion}

Most early-stage clinical trials with immunotherapy have shown promising results even in patients with heavily pretreated BTC. However, further large-scaled prospective studies are needed to verify the efficacy and identify predictive markers of immunotherapy.

\section{References}

1. Glimelius B, Hoffman K, Sjoden PO, Jacobsson G, Sellstrom H, et al (1996) Chemotherapy improves survival and quality of life in advanced pancreatic and biliary cancer. Annals of oncology : official journal of the European Society for Medical Oncology 7(6): 593-600.

2. Sharma A, Dwary AD, Mohanti BK, Deo SV, Pal S, et al. (2010) Best supportive care compared with chemotherapy for unresectable gall bladder cancer: a randomized controlled study. Journal of clinical oncology 28(30): 4581-4586.

3. Yonemoto N, Furuse J, Okusaka T, Yamao K, Funakoshi A, et al. (2007) A multi-center retrospective analysis of survival benefits of chemotherapy for unresectable biliary tract cancer. Japanese journal of clinical oncology 37(11): 843-851.

4. Ji JH, Kim YS, Park I, Lee SI, Kim RB, et al. (2017) Chemotherapy Versus Best Supportive Care in Advanced Biliary Tract Carcinoma: A MultiInstitutional Propensity Score Matching Analysis. Cancer research and treatment.

5. Valle J, Wasan H Fau - Palmer DH, Palmer Dh Fau - Cunningham D, 
Cunningham D Fau - Anthoney A, Anthoney A Fau - Maraveyas A, et al. Cisplatin plus gemcitabine versus gemcitabine for biliary tract cancer. $\mathrm{N}$ Engl J Med 362(14): 1273-1281.

6. Rao S, Cunningham D, Hawkins RE, Hill ME, Smith D, et al. (2005) Phase III study of 5FU, etoposide and leucovorin (FELV) compared to epirubicin, cisplatin and 5FU (ECF) in previously untreated patients with advanced biliary cancer. British journal of cancer 92(9): 1650-1654.

7. Glimelius B, Hoffman K Fau-Sjoden PO, Sjoden Po Fau - Jacobsson G, Jacobsson G Fau - Sellstrom H, Sellstrom H Fau - Enander LK, et al. (1996) Chemotherapy improves survival and quality of life in advanced pancreatic and biliary cancer. Annals of Oncology 7(6): 593-600.

8. Andre T, Tournigand C Fau - Rosmorduc O, Rosmorduc O Fau - Provent S, Provent S Fau - Maindrault-Goebel F, Maindrault-Goebel F Fau Avenin D, Avenin D Fau - Selle F, et al. (2004) Gemcitabine combined with oxaliplatin (GEMOX) in advanced biliary tract adenocarcinoma: a GERCOR study.Ann Oncol 15(9): 1339-1343.

9. Knox JJ, Hedley D, Oza A, Feld R, Siu LL, et al. (2005) Combining gemcitabine and capecitabine in patients with advanced biliary cancer: a phase II trial. Journal of clinical oncology 23(10): 2332-2338.

10. Koeberle D, Saletti P, Borner M, Gerber D, Dietrich D, et al. (2008) Patient-reported outcomes of patients with advanced biliary tract cancers receiving gemcitabine plus capecitabine: a multicenter, phase II trial of the Swiss Group for Clinical Cancer Research. Journal of clinical oncology 26(22): 3702-3708.

11. Riechelmann RP, Townsley CA, Chin SN, Pond GR, Knox JJ (2007) Expanded phase II trial of gemcitabine and capecitabine for advanced biliary cancer. Cancer 110(6): 1307-1312.

12. Andre T, Reyes-Vidal JM, Fartoux L, Ross P, Leslie M, et al. (2008) Gemcitabine and oxaliplatin in advanced biliary tract carcinoma: a phase II study. British journal of cancer 99(6): 862-867.

13. Harder J, Riecken B, Kummer O, Lohrmann C, Otto F, et al. (2006) Outpatient chemotherapy with gemcitabine and oxaliplatin in patients with biliary tract cancer. British journal of cancer 95(7): 848-852.

14. Kim YJ, Im SA, Kim HG, Oh SY, Lee KW, et al. (2008) A phase II trial of S-1 and cisplatin in patients with metastatic or relapsed biliary tract cancer. Annals of oncology 19(1): 99-103.

15. Kang MJ, Lee JL, Kim TW, Lee SS, Ahn S, et al. (2012) Randomized phase II trial of S-1 and cisplatin versus gemcitabine and cisplatin in patients with advanced biliary tract adenocarcinoma. Acta oncologica (Stockholm, Sweden) 51(7): 860-866.

16. Kim TW, Chang HM, Kang HJ, Lee JR, Ryu MH, et al. (2003) Phase II study of capecitabine plus cisplatin as first-line chemotherapy in advanced biliary cancer. Annals of oncology 14(7): 1115-1120.

17. Hong YS, Lee J, Lee SC, Hwang IG, Choi SH, et al. (2007) Phase II study of capecitabine and cisplatin in previously untreated advanced biliary tract cancer. Cancer chemotherapy and pharmacology 60(3): 321-328.

18. Ji JH, Song HN, Kim RB, Oh SY, Lim HY, et al (2015) Natural history of metastatic biliary tract cancer (BTC) patients with good performance status (PS) who were treated with only best supportive care (BSC). Japanese journal of clinical oncology 45(3): 256-260.

19. He S, Shen J, Sun X, Liu L, Dong J (2014) A phase II FOLFOX-4 regimen as second-line treatment in advanced biliary tract cancer refractory to gemcitabine/cisplatin. Journal of chemotherapy 26(4): 243-247.

20. Sasaki T, Isayama H, Yashima Y, Yagioka H, Kogure H, Arizumi T, et al. (2009) S-1 monotherapy in patients with advanced biliary tract cancer. Oncology $77(1): 71-74$.

21. Sasaki T, Isayama H, Nakai Y, Mizuno S, Yamamoto K, et al. (2012) Multicenter phase II study of S-1 monotherapy as second-line chemotherapy for advanced biliary tract cancer refractory to gemcitabine. Investigational new drugs 30(2): 708-713.

22. Zhu AX, Meyerhardt JA, Blaszkowsky LS, Kambadakone AR, Muzikansky A, et al. (2010) Efficacy and safety of gemcitabine, oxaliplatin, and bevacizumab in advanced biliary-tract cancers and correlation of changes in 18-fluorodeoxyglucose PET with clinical outcome: a phase 2 study. The Lancet Oncology 11(1): 48-54.

23. Iyer RV, Pokuri VK, Groman A, Ma WW, Malhotra U, et al. (2016) A Multicenter Phase II Study of Gemcitabine, Capecitabine, and Bevacizumab for Locally Advanced or Metastatic Biliary Tract Cancer. American journal of clinical oncology. Am J Clin Oncol.

24. Lubner SJ, Mahoney MR, Kolesar JL, Loconte NK, Kim GP, et al. (2010) Report of a multicenter phase II trial testing a combination of biweekly bevacizumab and daily erlotinib in patients with unresectable biliary cancer: a phase II Consortium study. Journal of clinical oncology 28(21): 3491-3497.

25. Bengala C, Bertolini F, Malavasi N, Boni C, Aitini E, et al. (2010) Sorafenib in patients with advanced biliary tract carcinoma: a phase II trial. British journal of cancer 102(1): 68-72.

26. El-Khoueiry AB, Rankin CJ, Ben-Josef E, Lenz HJ, Gold PJ, et al (2012) SWOG 0514: a phase II study of sorafenib in patients with unresectable or metastatic gallbladder carcinoma and cholangiocarcinoma. Investigational new drugs 30(4): 1646-1651.

27. Lee JK, Capanu M, O'Reilly EM, Ma J, Chou JF, et al. (2013) A phase II study of gemcitabine and cisplatin plus sorafenib in patients with advanced biliary adenocarcinomas. British journal of cancer 109(4): 915-919.

28. El-Khoueiry AB, Rankin C, Siegel AB, Iqbal S, Gong IY, et al. (2014) S0941: a phase 2 SWOG study of sorafenib and erlotinib in patients with advanced gallbladder carcinoma or cholangiocarcinoma. British journal of cancer $110(4)$ : 882-887.

29. Gruenberger B, Schueller J, Heubrandtner U, Wrba F, Tamandl D, et al. (2010) Cetuximab, gemcitabine, and oxaliplatin in patients with unresectable advanced or metastatic biliary tract cancer: a phase 2 study. The Lancet Oncology 11(12): 1142-1148.

30. Malka D, Cervera P, Foulon S, Trarbach T, de la Fouchardiere C, et al. (2014) Gemcitabine and oxaliplatin with or without cetuximab in advanced biliary-tract cancer (BINGO): a randomised, open-label, noncomparative phase 2 trial. The Lancet Oncology 15(8): 819-828.

31. Lee J, Park SH, Chang HM, Kim JS, Choi HJ, et al. (2012) Gemcitabine and oxaliplatin with or without erlotinib in advanced biliary-tract cancer: a multicentre, open-label, randomised, phase 3 study. The Lancet Oncology 13(2): 181-188.

32. Landskron G, De la Fuente M, Thuwajit P, Thuwajit C, Hermoso MA (2014) Chronic inflammation and cytokines in the tumor microenvironment. Journal of immunology research.

33. Yoshitomi M, Yutani S, Matsueda S, Ioji T, Komatsu N, et al. (2012) Personalized peptide vaccination for advanced biliary tract cancer: IL-6, nutritional status and pre-existing antigen-specific immunity as possible biomarkers for patient prognosis. Experimental and therapeutic medicine 3(3): 463-469.

34. Aruga A, Takeshita N, Kotera Y, Okuyama R, Matsushita N, et al. (2013) Long-term Vaccination with Multiple Peptides Derived from CancerTestis Antigens Can Maintain a Specific T-cell Response and Achieve Disease Stability in Advanced Biliary Tract Cancer. Clinical cancer research 19(8): 2224-2231.

35. Aruga A, Takeshita N, Kotera Y, Okuyama R, Matsushita N, et al. (2014) Phase I clinical trial of multiple-peptide vaccination for patients with advanced biliary tract cancer. Journal of translational medicine 12: 61 .

36. Ye Y, Zhou L, Xie X, Jiang G, Xie H, et al. (2009) Interaction of B7-H1 on intrahepatic cholangiocarcinoma cells with PD-1 on tumor-infiltrating $\mathrm{T}$ cells as a mechanism of immune evasion. Journal of surgical oncology 


\section{0(6): 500-504.}

37. Bang YJ, Doi T, Braud FD, Piha-Paul S, Hollebecque A, Razak et al. (2015) 525 Safety and efficacy of pembrolizumab (MK-3475) in patients (pts) with advanced biliary tract cancer: Interim results of KEYNOTE-028.
European Journal of Cancer 51: S112.

\begin{tabular}{|l} 
BIOMEDICAL \\
RESEARCHES
\end{tabular}$\quad \begin{aligned} & \text { Assets of Publishing with us } \\
& \text { - Global archiving of articles }\end{aligned}$

